\title{
Germanica
}

\section{Le temps suspendu : fil, bobine, écheveau - un parcours à travers quelques pièces de Lukas Bärfuss}

Hängende Zeit: Faden, Spule, Strang - ein Blick auf einige Stücke von Lukas

Bärfuss

Hanging time: an insight into some of Lukas Bärfuss's dramatic works

\section{Anne-Sophie Gomez}

\section{OpenEdition}

\section{Journals}

Édition électronique

URL : http://journals.openedition.org/germanica/2606

DOI : 10.4000/germanica.2606

ISSN : 2107-0784

\section{Éditeur}

Université de Lille

Édition imprimée

Date de publication : 30 juin 2014

Pagination : 159-174

ISBN : 9782913857339

ISSN : 0984-2632

\section{Référence électronique}

Anne-Sophie Gomez, «Le temps suspendu : fil, bobine, écheveau - un parcours à travers quelques pièces de Lukas Bärfuss », Germanica [En ligne], 54 | 2014, mis en ligne le 30 juin 2017, consulté le 06 octobre 2020. URL : http://journals.openedition.org/germanica/2606 ; DOI : https://doi.org/10.4000/ germanica.2606 


\title{
Le temps suspendu : fil, bobine, écheveau - un parcours à travers quelques pièces de Lukas Bärfuss
}

\author{
Anne-Sophie GOMEZ \\ CELIS / Université Blaise Pascal - Clermont-Ferrand
}

\section{Une écriture du déroulement}

\section{«Chronique d'une mort annoncée » : linéarité et chronologie}

La pièce Meienbergs Tod a été représentée pour la première fois en 2001. Elle retrace, à travers quelques épisodes jugés marquants par l'auteur, le déroulement de la vie de Niklaus Meienberg, jusqu'à la mort de l'écrivain et journaliste helvétique, dont le décès est d'emblée présenté comme l'issue de la pièce. En dépit de ruptures, notamment à travers les interventions métadramatiques des comédiens, un fil narratif et chronologique est clairement discernable. Dans la pièce intitulée Der Bus, laquelle donne à voir une partie du trajet en car d'Erika, pèlerin égarée, on ne trouve certes plus de découpage en actes, mais un marquage du déroulement chronologique, à travers des mentions allant de «Erstens » à « Fünftens ». La structure de la pièce est d'ailleurs d'emblée annoncée dans une mention en italique précisant le lieu de l'action et succédant à la liste de personnages :

Dans une forêt au milieu des montagnes, le long d'une route; plus tard à la station-service d'Anton; ensuite sur un plateau; enfin près 
d'un lieu qui ressemble au foyer de passage sur la grand-place de Częstochowa en Pologne 1.

Le trajet en car est certes entrecoupé par plusieurs arrêts, mais le conducteur du car, Hermann, insiste sur le fait que ce trajet doit se poursuivre, et il exhorte à plusieurs reprises les passagers à ne pas descendre de manière intempestive ou à ne pas différer plus longtemps le départ, comme si sa feuille de route était une sorte de métaphore du déroulement du spectacle, qu'il lui fallait respecter sans perdre de passagers/ personnages, à l'exception d'Erika, montée par erreur :

La grosse dame et Jasmin descendent de l'autocar.

HERMANN - Interdiction de descendre. Ceci n'est pas un arrêt régulier. Le trajet va se poursuivre sans plus tarder.

[...]

KARL descend du car.

HERMANN - Remontez immédiatement. Le trajet continue. Remontez ${ }^{2}$.

La pièce Die sexuellen Neurosen unserer Eltern s'articule elle aussi autour du déroulement chronologique de scènes numérotées de 1 à 35 . En 2006, dans Alices Reise in die Schweiz, Lukas Bärfuss documente le voyage « initiatique » du personnage éponyme, de sa prise de décision à la mise en œuvre de celle-ci.

L'auteur ne renonce donc pas à la linéarité, mais interrompt régulièrement celle-ci, sans toutefois la briser. Lukas Bärfuss semble en effet accorder une importance particulière à l'ordre des événements. Et s'il s'autorise des ellipses - comme lorsqu'il aligne des fragments de la vie de Meienberg ${ }^{3}$ - il évite en revanche les analepses et les prolepses. Comme le souligne Peter Michalzik dans son article «Dramen für ein Theater ohne Drama. Traditionelle neue Dramatik bei Rinke, von Mayenburg, Schimmelpfennig und Bärfuss » : «Bärfuss s’appuie sur les éléments du drame ouvert, éclaté, mais ce faisant il associe si habilement les fragments que, du moins par instants, ils donnent l'illusion de former une vitre ou un vase intacts $»^{4}$. Et si l'on peut parler, pour

1. - Lukas Bärfuss, Stücke, Göttingen : Wallstein Verlag, 2005, p. 132. «In einem Wald in den Bergen, an einer Straße; später bei Antons Tankstelle; danach auf einer Hochebene; schließlich an einem Ort, ähnlich dem Durchgangsheim am Glowny Rynek in Tschenstochau, Polen ». C'est nous qui soulignons et qui traduisons.

2. - Die Dicke und Jasmin steigen aus dem Bus. / HERMANN Nicht aussteigen. Hier kein ordentlicher Halt. Die Fahrt wird umgehend fortgesetzt. [...] KARL tritt aus dem Bus. / HERMANN Einsteigen. Die Fahrt wird fortgesetzt. Einsteigen. Ibid, p. 146 et 156.

3. - Les différents épisodes représentés se situent respectivement en 1964, 1971, $1980,1984$.

4. - « Bärfuss arbeitet mit den Elementen des offenen, zersplitterten Dramas, er setzt die Splitter gleichzeitig aber so geschickt aneinander, dass sie mindestens zeitweise 
qualifier Meienbergs Tod, de drame épisodique ( Stationendrama »), c'est non seulement parce que les différents épisodes sont liés entre eux grâce au personnage éponyme Meienberg, mais aussi parce que le déroulement chronologique - fût-il elliptique et chaotique - de ce parcours, de la naissance jusqu'à la mort, lui confère de la cohérence. La pièce intitulée Amygdala 5 illustre elle aussi la conception qu'a Lukas Bärfuss du fragment - fragment qui, selon lui, ne met pas en péril sinon l'intégrité, du moins l'intégralité de l'ensemble, ainsi qu'en témoigne le sous-titre, pour le moins oxymorique : «Fragments complets d'une ville incomplète ${ }^{6}$.

\section{Fils, câbles, ficelles, bobines : des métaphores programmatiques}

$\mathrm{Au}$ déroulement narratif fait écho, chez Bärfuss, le déroulement de bobines de pellicule : chaque pièce se termine ainsi par la mention «Fin de la bobine », en français dans le texte, inscrivant le spectacle dans un paradigme cinématographique. Cela n'est certes pas chose rare au cours des années 2000, ainsi que l'a souligné Anne Monfort dans un article où elle interroge les interactions entre spectacle vivant, langage scénique, et cinéma :

La référence cinématographique [...] fonctionne également de façon plus structurante et conditionne l'écriture même de certains textes. [...] Actuellement, des auteurs de théâtre utilisent le cinéma et la vidéo à la fois comme citation, comme référence et comme fonction structurante de l'écriture ${ }^{7}$.

Le Suisse Lukas Bärfuss utilise le cinéma comme matériau : l'une de ses expériences avec sa compagnie « 400asa » a été de recopier phonétiquement la Médée de Lars von Trier et de donner ce matériau aux

wie eine unzerbrochene Scheibe oder ein unzerbrochener Krug wirken. » Michalzik, Peter: «Dramen für ein Theater ohne Drama. Traditionelle neue Dramatik bei Rinke, von Mayenburg, Schimmelpfennig und Bärfuss ». In: Stefan Tigges (dir.): Dramatische Transformationen. Zu gegenwärtigen Schreib- und Aufführungsstrategien im deutschsprachigen Theater, Bielefeld, transcript, 2008, p. 40. Peter Michalzik parle, quelques lignes plus loin, pour qualifier l'écriture de Bärfuss par rapport aux autres auteurs du corpus, « d'effets kaléidoscopiques irritants » (« irrientierend [e] kaleidoskopartig [e] Effekt [e] $»)$,ibid., p. 41.

5. - Texte publié en 2007, première représentation en 2009 au Thalia Theater de Hambourg.

6. - «Vollständige Fragmente einer unvollständigen Stadt ». Bärfuss, Lukas : Stücke, Göttingen : Wallstein Verlag, 2007, p. 115.

7. - Anne Monfort, « Theâtre et langage cinématographique : correspondance ou superposition des signes? Autour de quelques dramaturges contemporains : Fritz Kater, Lukas Bärfuss, Falk Richter». In : Brender, Edwige, Hausbei, Kerstin et al. (dir.) : A la croisée des langages. Texte et art dans les pays de langue allemande, Paris, Presses Sorbonne nouvelle, 2006, p. 209. 
comédiens. Les pièces de cet auteur s'achèvent par « fin de la bobine ». [...] C'est l'écriture filmique proprement dite qui est intégrée dans un code de représentation théâtral, comme matériau stylistique ou sonore ${ }^{8}$.

Le nom de la compagnie « 400asa », fondée en 1998 par Samuel Schwarz, Lukas Bärfuss et Udo Israel, est à cet égard révélateur de l'importance prise par l'image aux yeux de Bärfuss puisque l'expression « 400 ASA » renvoie à la mesure de la photosensibilité d'une pellicule ou d'un capteur numérique. Toutefois, si la référence au cinéma semble très forte, c'est moins esthétiquement que poétologiquement : en effet, c'est encore une fois le mouvement de déroulement, de dévidement de la bobine qui semble intéresser l'auteur, davantage que le potentiel de souplesse narrative et temporelle que renferme le genre cinématographique. Dans son étude sur le théâtre contemporain, Gerda Poschmann explique le fréquent recours de la jeune génération de dramaturges à des procédés cinématographiques par la grande familiarité du public avec de tels procédés :

Les dramaturges introduisent différents niveaux narratifs [...] en empruntant au genre cinématographique ses procédés (analepse, montage, découpage) [...]. Des monologues («intérieurs ») à interpréter sous forme de pensées rendues audibles et l'insertion de scènes mises en perspective - qui pour certaines convoquent des événements passés - étayent la fiction par le fréquent recours aux habitudes visuelles d'un public familier de l'esthétique cinématographique. Il en est de même pour les éruptions fantastiques ou absurdes au sein d'une fiction dramatique qui se présente au premier abord comme réaliste 9 .

Or Bärfuss, en rendant hommage à la projection d'avant l'ère numérique, c'est-à-dire à l'artisanat plus qu'à l'artifice ou à l'effet spécial, se singularise par rapport au travail de nombre des auteurs de sa génération. Il ne s'agit pas non plus pour lui d'utiliser la projection comme loupe ni comme insert à visée distanciatrice.

Dans Meienbergs Tod, Lukas Bärfuss assigne d'autre part au temps le rôle de fil conducteur. Au-delà du déroulement de l'existence du personnage éponyme, le dramaturge donne aussi concrètement à voir la durée

8. - Ibid., p. 211 .

9. - « Epische Ebenen werden [...] in Anlehnung an Techniken des Films (Rückblende, Montage, Schnitt) eingesetzt [...]. Als hörbar gemachte Gedanken zu verstehende ("innere") Monologe und eingeschobene, perspektivisch geprägte, teils erinnernde Szenen unterstützen im Gegenteil die Fiktion oft unter Rekurs auf die Zuschaugewohnheiten eines von der Filmästhetik geprägten Publikums. Ähnliches gilt für phantastische oder absurde "Ausbrüche" aus einer sich zunächst realistisch präsentierenden Stückfiktion [...] ». Gerda Poschmann, Der nicht mehr dramatische Theatertext: aktuelle Bühnenstücke und ihre dramaturgische Analyse. Tübingen: Niemeyer, 1997, p. 68. 
réelle de la représentation, matérialisée par une pendule suspendue au bout d'un câble au centre de la scène ${ }^{10}$ - comme si à la tension dramatique venait se substituer une tension mécanique. Dans sa critique de la pièce, Beat Mazenauer parle de « corset temporel très explicite de 115 minutes (la pendule décompte le temps à l'arrière-plan) ${ }^{11}$. La représentation, dès lors, fonctionne sur le principe du compte à rebours. Le déroulement en temps réel d'une durée arbitraire, préalablement fixée et intangible, du fait qu'il est vécu à la fois par les acteurs et par les spectateurs, fonde une expérience partagée, dont le ciment n'est plus la narration ni l'identification: Hans-Thies Lehmann parle, à propos de ce type de temporalité, de "temps réel vécu comme une situation partagée [entre le public et la scène] ${ }^{12}$. Cela est d'autant plus vrai chez Bärfuss, dans le cas de Meienbergs Tod notamment, que la pièce comporte la mention «Époque : actuelle » ${ }^{13}$, or la pièce ne peut être plus «contemporaine » de sa représentation puisqu'elle est jouée en temps réel. Soulignons d'ailleurs que le temps se trouve non seulement au centre de la scène, mais aussi au centre de la pièce. Les références au temps qui s'écoule sont d'ailleurs de plus en plus nombreuses vers la fin de la pièce, et créent une nouvelle forme de tension, de nature extradiégétique et métadramatique, qui n'est pas sans être teintée d'une intention ludique ${ }^{14}$. Le public se demande ainsi si les acteurs vont réussir à respecter le temps qui leur est imparti, et à clore la pièce conformément à ce qui avait initialement été annoncé. Le chœur veille tout particulièrement à rappeler cette contrainte temporelle, contribuant à accroître celle-ci :

10. - « On aperçoit la scène vide avec, en son centre, une pendule suspendue, qui décompte les minutes à partir de 115 ». «Man erkennt die leere Bühne, in deren Mitte eine Uhr hängt, die sofort von 115 gegen Null zu laufen beginnt ». Lukas Bärfuss, Stücke (2005), p. 9.

11. - « [...] klar umrissenen Zeitkorsetts von 115 Minuten (die Uhr läuft im Hintergrund $a b)$ ». Beat Mazenauer: « Jeder Mensch ist korrupt ». URL: http://www. culturactif.ch/livredumois/juillet05barfuss.htm [Consulté le 19.11.13].

12. - « Realzeit als gemeinsam durchlebt[e] Situation». Hans-Thies Lehmann, Postdramatisches Theater, Frankfurt am Main: Verlag der Autoren, 1999, p. 327.

13. - "Ort: Die Bühne eines Theaters.

Zeit: Gegenwart». Lukas Bärfuss, Stücke (2005), p. 8.

14. - Peter Michalzik écrit ainsi, à propos de l'effet provoqué par les pièces de Lukas Bärfuss, après avoir comparé les « scènes » qui composent les pièces de Bärfuss à des « syntagmes dramatiques » («theatrale Syntagmen ») : « Ce qui en résulte n'est pas de la pure théorie, mais une reconquête du jeu. L'auteur va-t-il parvenir à réunir les fragments pour en faire un tout convaincant ? ». «Was hier [...] am Ende steht, ist nicht graue Theorie, sondern eine Wiedergewinnung des Spiels. Bekommt der Autor es hin, die Splitter zu einem überzeugenden Ganzen zusammenzubringen?». «Dramen für ein Theater ohne Drama. Traditionelle neue Dramatik bei Rinke, von Mayenburg, Schimmelpfennig und Bärfuss », in: op. cit., p. 41. 
Très révéré public,

[...] hélas

Vous le voyez le temps ne va pas suffire

[...]

Et pendant que nous racontons ce qui pourrait être montré si le temps

suffisait

ce temps s'écoule et il ne nous en reste

guère pour honorer notre partie du contrat

et pour vous montrer ce que vous avez payé pour voir

ce pour quoi vous êtes

venus ici. $[\ldots]^{15}$

Les acteurs ne peuvent que relayer ce constat, et dresser le bilan en demi-teinte de leur prestation. Malgré tout, le contrat est rempli, Meienberg est bien mort, et le public en aura pour son argent :

\footnotetext{
HELLA

$[\ldots]$
}

Elle regarde la pendule.

Le temps file, mon ami, d'autres viennent après nous,

Ils arrivent déjà.

$[\ldots]$

Hans prend le pouls de Daniel. Puis il fait un signe de la tête.

Hella - Au moins, on a eu suffisamment de temps pour cela.

J'aurais dû prononcer une jolie phrase de conclusion. Tant pis, je la

dirai la prochaine fois.

La pendule affiche zéro.

La scène est plongée dans l'obscurité 16 .

Die sexuellen Neurosen unserer Eltern comporte aussi, formulée dans une indication scénique, une métaphore du temps qui s'étire métaphore basée sur la matérialisation du temps en fils, renforçant l'impression que le motif du fil et de la suspension occupe, plus qu'une fonction pratique, une fonction poétologique :

15. - « Hochverehrtes Publikum! / [...] Leider / Sie sehen es wird die Zeit nicht mehr reichen. / [...] / Und während wir hier also erzählen / Was zu sehen wäre würde die Zeit noch / reichen verstreicht diese Zeit und es bleibt uns / kaum noch Zeit unseren Teil des Vertrags zu / erfüllen und Ihnen zu zeigen was zu sehen / Sie bezahlt haben und weswegen Sie hier / Hergekommen sind. » Bärfuss, Lukas: Stücke (2005), p. 62-63.

16. - « HELLA [...] / Sie blickt auf die Uhr. / Die Zeit fliegt, mein Freund, es kommen andere nach uns, / sie sind schon da. / [...] / HANS fühlt Daniels Puls. Dann nickt er. / HELLA Immerhin, dafür hat die Zeit gereicht. Ich hätte einen schönen Schlußsatz gehabt. Na, ich sage ihn beim nächsten Mal. / Die Uhr ist bei Null angelangt. Nun wird es dunkel », ibid., p. 69. 
34. CHEZ LE MÉDECIN. LE TEMPS S'ÉTIRE AU POINT DE PRODUIRE DE LONGS FILS, JUSQU'À ROMPRE ${ }^{17}$.

Enfin, on pourrait ajouter que Bärfuss donne parfois à voir les ficelles de la représentation, c'est-à-dire ce qui relève habituellement des coulisses, comme la panne technique qui survient dans Meienbergs Tod et modifie prétendument le cours de la représentation.

Fils, ficelles, câbles et bobines excèdent donc largement le statut de simples accessoires. Ils indiquent combien le tissage d'une trame narrative et dramatique reste fondamental pour l'auteur, mais nous y reviendrons.

\section{Une stratégie de brouillage générique}

\section{Indétermination}

La narration, dans Meienbergs Tod notamment, oscille constamment entre emprunts biographiques et éléments fictionnalisés. La dimension narrative est assumée par le chœur, composé des acteurs de la pièce et qui, dès le début de la représentation, s'adresse solennellement au public dans un récitatif afin de présenter le sujet de la pièce, qui ne sera finalement pas tant la mort de Meienberg que des scènes tirées de la vie de celui-ci :

CHEUR

Très révéré public,

Vous êtes venus

Assister ce soir à la mort du journaliste

Meienberg à partir

De quelques scènes dont on supposera

Qu'elles sont tirées de sa vie ${ }^{18}$.

Rapidement, le chœur sème le trouble sur l'authenticité des scènes qui vont suivre :

Nous vous montrerons une vie qui remonte à une époque si ancienne

Que l'on ne sait plus

S'il s'agit d'une histoire vraie ou bien inventée

Par quelqu'un qui s'ennuyait ou qui l'a volontairement imaginée ${ }^{19}$.

17. - « 34. BEIM ARZT. DIE ZEIT DEHNT UND DEHNT SICH ZU LANGEN FÄDEN, BIS SIE SCHLIESSLICH REISST. »Ibid., p. 123.

18. - «CHOR / Hochverehrtes Publikum! / Sie sind gekommen um / Heute Abend den Tod des Journalisten / Meienberg zu sehen anhand / Einiger Szenen von denen man annimmt / Sie seien gegriffen aus seinem Leben. » Ibid., p. 9.

19. - «Ein Leben aus so alter Zeit zeigen wir / daß wir nichts mehr wissen / ist die Geschichte auch wahr oder etwa erfunden / Von einem aus Langeweile oder Absicht. » Ibid., p. 9. 
Si la pièce s'appuie bien sur un fond(s) biographique, l'auteur veille à ce qu'elle ne bascule pas du côté du genre du « biopic » et de la reconstitution zélée. D'autre part, les comédiens sont désignés par leur prénom - fictif - sans que soit précisé quel rôle ils interprètent, car ce rôle est susceptible d'être échangé en cours de représentation. L'auteur opère ainsi un téléscopage entre le plan dramatique et le plan extradiégétique, puisque la distribution des rôles apparaît rapidement comme une source de tension - certes fictive et scénarisée - entre les comédiens-personnages.

La pièce Der Bus comporte quant à elle la mention générique "Schauspiel », inscrivant la pièce dans la tradition dramatique et fictionnelle. Néanmoins, son ancrage générique reste incertain : s'agit-il d'une pièce religieuse, d'une passion, ainsi que son sous-titre ( «L'étoffe d'une sainte » ${ }^{20}$ ) le laisserait entendre ? Ou bien d'un roadmovie, qui se déroule sous les yeux du spectateur jusqu'à l'ultime rotation de la pellicule (« fin de la bobine ») ? Sur son site Internet, la compagnie «400asa » qualifie pour sa part la pièce en ces termes : «thriller protestant avec une imagerie catholique »21. Quant au titre de la pièce Alices Reise in die Schweiz, il renvoie à un voyage initiatique paradoxal, puisque situé non pas au seuil de la vie d'adulte, comme c'est habituellement le cas dans le genre du roman de formation, mais au seuil de la mort. Néanmoins, on ne sait si on doit interpréter cette référence comme unilatéralement parodique, dans la mesure où Alice effectue malgré tout d'ultimes "découvertes » à l'occasion de son voyage, partagée entre attentes, déconvenues et dégoût :

ALICE - Un si beau pays. La Suisse. Chez nous les gares sont des trous. Mais ici. Tellement conviviales. On aimerait carrément habiter dedans. Et ces plates-bandes de fleurs partout. Super. Gustav. Je suis un peu déçue.

GustaV - Ah bon.

ALICE - Je suis passée devant deux sex-shops, et partout ces montagnes d'ordures. C'est un mauvais quartier, ce quartier ${ }^{22}$.

En choisissant pour sous-titre de sa pièce « Szenen aus dem Leben des Sterbehelfers Gustav Strom » («Scènes de la vie de l'euthanasiste

20. - «Das Zeug einer Heiligen ».

21. - « protestantische [r] Thriller mit katholischer Ikonografie ». URL : http://400asa.ch/theater/aktuell/bus [consulté le 03.12.13].

22. - «ALICE So ein schönes Land. Die Schweiz. Bei uns sind die Bahnhöfe ja Löcher. Aber hier. So menschenfreundlich. Da möchte man direkt drin wohnen. Und diese Blumenrabatte überall. Toll. Gustav. Ich bin ein bisschen enttäuscht. / GUSTAV So. / ALICE Ich kam an zwei Sexshops vorbei, und überall diese Abfallberge. Das ist ein schlechtes Viertel, dieses Viertel. » Bärfuss, Lukas : Stücke (2007), p. 48. Traduction française de Hélène Mauler et René Zahnd. Paris: L'Arche, 2011, p. 61. 
Gustav Strom »), Lukas Bärfuss opte clairement pour une stratégie de brouillage des repères du spectateur : outre la référence à Lewis Carroll, l'opposition entre les termes «Leben » (la vie) et "Sterbehelfer » (l'euthanasiste), la présence d'un deuxième prénom disjoint de celui du personnage éponyme ainsi que l'allusion à une esthétique fragmentaire ( «Szenen ») ne permettent pas au lecteur/spectateur de se faire une idée du genre de la pièce qu'il s'apprête à découvrir.

\section{Distanciation farcesque}

Dès le sous-titre, le spectateur est informé de l'hybridité générique de la pièce Meinbergs Tod, laquelle fait certes nécessairement écho, pour l'amateur de théâtre, au Dantons Tod de Büchner, mais que l'auteur qualifie de « Groteske » («Farce ») ${ }^{23}$. C'est ce que confirment par exemple les querelles entre les comédiens, lesquelles font figure de chamailleries dignes d'une cour de récréation, ou encore les qualificatifs dont s'affuble le personnage éponyme, farcesques du fait de la créativité et de l'audace verbale débridées dont ils témoignent, mais aussi du fait de leur accumulation:

DANIEl comme pris dans une embuscade : Un tas de fumier, voilà ce que je suis, un abruti, un toubib, un empoté, une truie à saigner, dans le meilleur des cas et parfois le dimanche il m'arrive d'être une bête, une tête d'œuf, un pétochard, un branleur, un crétin $[\ldots]^{24}$.

Au-delà de cette énumération - sorte de bouquet verbal final de Meienberg/Daniel - certaines scènes de la pièce se révèlent elles aussi particulièrement triviales et contrastent vivement avec la gravité et la solennité véhiculées tant par le titre que par les propos, syntaxiquement et lexicalement très soutenus voire légèrement désuets, du chœur. C'est notamment le cas lors de «l'épiphanie d'Hamlet»: le personnage de Meienberg se trouve ainsi brusquement stoppé au milieu d'une pulsion sexuelle par une apparition «shakespearienne » qui mue son envie en inspiration - ou plutôt en accablement - métaphysique :

DANIEL $s$ 'interrompt brutalement en plein lutinage. Mon Dieu !

RUTH - Qu'y a-t-il ?

DANIEL - C'est épouvantable.

RuTH - Continue.

DANIEL - Je ne peux pas.

Il se retire et s'assoit sur le sol. D'un air apathique :

23. - Lukas Bärfuss, Stücke (2005), p. 7.

24. - «DANIEL wie aus einem Hinterhalt: Ein Misthaufen bin ich, ein Fetzenschädel, ein Nillenflicker, ein Nöler, eine Blutsau, bestenfalls und an Sonntagen hin und wieder ein Schnulztier, ein Eierkopf, ein Angstarsch, ein Flachwichser, ein Blechschwätzer [...]», ibid., p. 61. 
Hamlet vient de m'apparaître une nouvelle fois. Cela m'arrive de temps à autre.

RUTH - Tu plaisantes ! Je vais bien m'occuper de toi.

DANIEL - Non, laisse-moi. Tu ne comprends pas. Ça me prend, c'est métaphysique

Silence.

Ne voudrais-tu pas être Ophélie ? Mon Ophélie?25

Le personnage éponyme apparaît ainsi comme un anti-héros exposé au grotesque. Et lorsque Meienberg/Daniel se décide à mourir, ses partenaires ne se privent pas d'exhortations réjouies («Es-tu prêt ? C'est l'heure de mourir maintenant. [...] Allons. Courage, c'est le moment ${ }^{26}$ ) ni de commentaires incisifs renforcés par des indications scéniques tout aussi ironiques:

Il regarde Eva d'un œil vide, de la même manière que Jeanne d'Arc sur son bûcher a regardé le ciel ${ }^{27}$.

La volonté de distanciation - ni « biopic », ni hagiographie transparaît d'ailleurs jusque dans la mise en scène puisque lors de la Première, les personnages apparaissent en perruques blanches évidemment anachroniques:

Le tandem Bärfuss/Schwarz aligne toute une batterie de techniques destinées à créer de la distance. Ils qualifient leur pièce de farce et sur ce point ils n'ont pas tort. Ils affublent les comédiens de costumes baroques et de perruques poudrées (décors et costumes Esther Schmid) et font de la mort de Meienberg du théâtre au théâtre ${ }^{28}$.

25. - «DANIEL hält im Vögeln plötzlich inne. O Gott! / RUTH Was ist? / DANIEL Schrecklich. / RUTH Mach weiter. / DANIEL Ich kann nicht. / Er zieht sich zurück und setzt sich auf den Boden. / Apathisch: Jetzt ist mir wieder Hamlet erschienen. Das habe ich manchmal. / RUTH Ach komm. / Ich mache es dir ganz schön. / DANIEL Nein, laß mich. Das verstehst du nicht. Das packt mich metaphysisch./ Schweigen. / Möchtest du nicht die Ophelia sein? Meine Ophelia ? » Bärfuss, Lukas: Stücke (2005), p. 34.

26. - «Bist du bereit ? Jetzt geht es an Sterben. [...] Also los. Nur aufrecht !», ibid., p. 64.

27. - «Mit leerem Blick, wie die heilige Johanna auf dem Scheiterhaufen den Himmel, so blickt er die Eva an ». Ibid., p. 61.

28. - «Das Tandem Bärfuss/Schwarz fährt eine ganze Batterie Distanz schaffender Verfremdungstechniken auf. Sie nennen ihr Stück eine Groteske und haben darin nicht Unrecht. Die Schauspieler lassen sie in Barockkostüme samt Weisshaar-Perücken stecken (Bühne und Kostüme Esther Schmid) und zeigen Meienbergs Tod als Theater auf dem Theater [...] ». « Die Leiche ist nicht tot. Meienbergs Tod in Basel uraufgeführt ». Neue Zürcher Zeitung, 23.04. 2001. Plusieurs extraits de cette mise en scène sont disponibles sur le site de la compagnie « 400asa ». URL: http://400asa.ch/theater/archiv/meienberg/ index.php [consulté le 03.12.13]. 
L'objectif de Bärfuss semble être de déshéroïser ses personnages, de faire affleurer leurs failles, celles qui précisément les rendent humains. Le décalage entre le commentaire du chœur et ce à quoi le public vient d'assister ( « La mort du journaliste Niklaus Meienberg, cruelle, mais exemplaire en son genre, et qui marqua la fin du siècle écoulé » ${ }^{29}$ ) participe de ce processus de déshéroïsation. En ce sens, nous pouvons dire que la conclusion du chœur est tout à fait programmatique :

[...] Que celui qui n'a que deux mains

Quitte sans plus tarder la salle. Aux autres

Nous souhaitons, outre l'édification intellectuelle,

Bien du plaisir ${ }^{30}$.

Lukas Bärfuss rappelle dans ce passage l'importance fondamentale du divertissement. Ce point de vue explique la co-présence d'éléments sérieux et d'éléments farcesques ou parodiques. Ces derniers sont aisément identifiables dans la discussion « scientifique » entre deux caricatures de germanistes concernant le sujet de thèse de l'un d'eux :

LE PLUS ÂGÉ - J'ai soutenu ma thèse sur « Le rôle des légumes dans les expressions idiomatiques allemandes ».

LE PLUS JEUNE - Un sujet fort intéressant, en effet et un vaste chantier.

LE PLUS ÂGÉ - En effet. Cela se trouve déjà chez Luther : la vie est aussi périssable que l'herbe.

LE PLUS JEUNE - L'herbe fait partie des légumes? Je ne savais pas !31

Le recours à des éléments parodiques et grotesques permet d'établir un contraste entre la gravité du sujet (la vie chaotique de Meienberg et son choix du suicide) et sa relativisation triviale, laquelle fait office de soupape de sécurité et permet au spectateur de se divertir. À la lecture des pièces de Lukas Bärfuss, il semble en effet que le rôle du théâtre soit, aux yeux du dramaturge, de proposer une réflexion dépassionnée qui sera amenée à se poursuivre au terme du cheminement dramatique - la « fin de la bobine » ne signifiant pas nécessairement la fin du film, mais éventuellement le basculement vers un autre projecteur.

29. - « Den grausamen, / in seiner Art jedoch exemplarischen Tod des / Journalisten Niklaus Meienberg mit welchem / das vergangene Jahrhundert zu Ende ging ». Bärfuss, Lukas: Stücke (2005), p. 63.

30. - « $[\ldots]$ Wer nicht vier Hände hat / der verlasse jetzt besser den Saal. Den anderen / wünschen wir neben geistiger Erbauung / recht viel Vergnügen », ibid., p. 613.

31. - «DER ÄLTERE Ich habe über „Die Rolle des Gemüses in der deutschen Idiomatik » promoviert. / DER JÜNGERE Ein hochinteressantes Thema, allerdings, und ein weites Feld. / DER ÄLTERE Allerdings. Man findet das schon bei Luther: Alles Leben vergeht wie Gras. / DER JÜNGERE Gras zählt man zum Gemüse? Das wußte ich nicht! », ibid., p. 45. 


\section{Les interruptions et leur fonction poétologique}

Dans une logique similaire, Lukas Bärfuss entrecoupe volontairement la narration de digressions, parfois très matérielles - à l'instar des doléances portant sur le salaire des comédiens, sur l'insatisfaction de ces derniers face à la distribution ou aux lieux des représentations ${ }^{32}$. De telles digressions soulignent la fictionnalité de ce qui est représenté, tout comme celle de la représentation. Pour Anne Monfort, ce procédé, caractéristique de ce qu'elle nomme l'écriture " néodramatique », contribue au « désamorçage ${ }^{33}$ de l'illusion représentative :

[La] présence concomitante de différents degrés de fiction était déjà un trait du thêâtre postdramatique : la situation thêâtrale est souvent assumée comme telle, les acteurs la dénoncent, s'adressent directement au public, brisent le quatrième mur. Deux degrés de réel cohabitent, à savoir le réel de la situation théâtrale et parfois aussi une fiction dramatique. [...] L'irruption de la diégesis dans la mimésis théâtrale fait coexister deux états : la situation théâtrale et la fiction intra-théâtrale. [...] C'est l'acteur qui est en charge de cette bascule d'un état à un autre et qui interroge la représentation ${ }^{34}$.

Nous soulignons toutefois que la digression possède, chez Lukas Bärfuss, une fonction d'interruption et non de rupture, dans la mesure où le fil conducteur (en l'occurrence, dans Meienbergs Tod, la contrainte temporelle) vient rappeler aux comédiens la raison d'être de leur présence sur scène. Hormis sous la forme du chœur, les comédiens ne s'adressent pas directement au public, mais ils discutent de celui-ci en sa présence, notamment de ses goûts en matière dramatique ( HELLA - La peur, c'est bien. Le public aime bien la peur ${ }^{35}$ ). L'interruption, qu'elle soit grotesque ou faussement métadramatique (les comédiens réfléchissent certes à leur métier et à leurs rôles, mais ils s'égarent dans des chamailleries puériles), permet paradoxalement au spectateur de demeurer conscient de la progression du spectacle, de son déroulement. Ainsi, il ne sera pas surpris lorsque la pièce s'achèvera - et c'est aussi le sens du dispositif de compte à rebours. Le dramaturge ne veut pas piéger son public dans une histoire, et veille à ne pas susciter de tension dramatique. Lorsque celle-ci se dessine à l'horizon,

32. - Voir Lukas Bärfuss, Stücke (2005), p. 15.

33. - Anne Monfort, «Après le postdramatique : narration et fiction entre écriture de plateau et théâtre néo-dramatique », Trajectoires [en ligne], 3 | 2009, mis en ligne le 16 décembre 2009, URL : http://trajectoires.revues.org/392 [consulté le 02.12.2013].

34. - Ibid.

35. - « HELLAAngst ist gut. Das Publikum mag Angst ». Bärfuss, Lukas : Stücke (2005), p. 17. 
elle se trouve aussitôt minée par un intertitre, «Alice meurt», («ALICE STIRBT ») ou une indication scénique annonçant ce qui va suivre.

\section{Les pièces de Lukas Bärfuss, véritables écheveaux intertextuels}

Si les bobines de Bärfuss se déroulent jusqu'au bout et sans incident majeur, il existe toutefois dans les pièces du dramaturge helvétique des aspects moins linéaires, à l'instar de l'enchevêtrement des références intertextuelles, qui forment de véritables écheveaux.

\section{Multiplicité et éclectisme des références}

Ce qui frappe d'emblée à la lecture des pièces de Lukas Bärfuss, c'est incontestablement l'éclectisme de leur arrière-plan intertextuel. Celui-ci peut prendre, assez classiquement, la forme de références intertextuelles, comme dans le cas de Büchner ou de Lewis Carroll. Parfois, il s'agit de convocations, comme dans le cas de Pablo Neruda qui, dans la distribution de Meienbergs Tod, apparaît comme l'essence du poète chilien : « le poète chilien » (« der chilenische Dichter »), sans que l'on puisse savoir quel traitement lui sera réservé, sachant qu'il appartient, au même titre que «le cheval » ou « les deux germanistes » évoqués plus haut, à la catégorie des personnages épisodiques (« episodische Rollen ») ${ }^{36}$. Concernant Shakespeare, nous avons déjà mentionné les références à Hamlet et à Ophélie, références que le contexte immédiat rend particulièrement irrévérencieuses. Parfois encore, l'allusion passe par des exergues là encore pour le moins éclectiques, d'Euclide pour Die Probe à Will Oldham, auteur compositeur et interprète américain de la génération de Bärfuss, pour Der Bus. Par-là, Lukas Bärfuss indique son souhait de s'inscrire dans la tradition littéraire, mais sans que celleci constitue un poids inhibant. Son objectif semble moins de vouloir déboulonner, provoquer ou choquer que de chercher, une nouvelle fois, à brouiller les repères du public et ainsi à élargir tant l'horizon réflexif de celui-ci que son potentiel critique par le biais d'une esthétique moins déférente que déceptive. L'auteur ne cherche pas non plus à établir de cohérence au sein des références qu'il énonce, le but n'étant nullement que le public parvienne à discerner une sorte de panthéon personnel du dramaturge. Les héros, nous l'avons vu avec Meienberg, sont des gens ordinaires, le plus souvent réduits à leur prénom (Nora, Erika).

\section{Derrière les fils, le canevas}

Le paradigme d'écriture de Lukas Bärfuss n'est pas selon nous celui du collage postmoderne, mais bien plutôt celui d'une tapisserie, qui

36. - Ibid., p. 8 . 
laisserait entrevoir son canevas. Une trame narrative reste certes identifiable, mais elle est savamment dépouillée, réduite à quelques bribes, à des repères spatio-temporels et onomastiques élémentaires permettant de situer ce qui se joue sur scène. Et ce sont des fils autres que les fils diégétiques qui s'insinuent alors dans les interstices ménagés par l'auteur. Ainsi le temps écoulé entre deux épisodes de la vie de Meienberg laisse-t-il deviner l'agitation du monde, par exemple le mouvement de protestation de $1968^{37}$. Dans Der Bus, c'est à une mise au jour de la méfiance, de l'individualisme contemporain et du réflexe d'agression voire d'exclusion que procède l'auteur. Quant à la pièce Die sexuellen Neurosen unserer Eltern, elle donne à voir la différence et la peur que celle-ci suscite. Toutefois, la narration n'en disparait pas pour autant, elle ne se dissout pas dans le questionnement sociétal ; la trame narrative, matérialisée par l'allusion à la bobine de film, reste la base de chacune des pièces. Car si la réflexion initiée par la narration finit par excéder celle-ci, c'est en la prolongeant, une fois la bobine entièrement dévidée, bien plutôt qu'en la saturant.

\section{Entre les interstices...}

Interrogé sur le sujet de l'implication politique de la littérature suisse contemporaine, Lukas Bärfuss affirme la nécessité pour l'écrivain de trouver sa propre orientation, plutôt que de vouloir imiter la position d'un Max Frisch. Selon lui, la littérature n'a pas à être politique (« La littérature n'a pas à satisfaire d'attentes, quelles qu'elles soient ${ }^{38}$ ) et l'écrivain n'a d'autre responsabilité à porter que celle de son œuvre. La littérature peut poser des questions, mais n'a pas à en fournir les réponses. Elle est toutefois parfaitement indissociable de la société, qui l'alimente, thématiquement et narrativement mais aussi concrètement, à travers le lectorat ou le public ${ }^{39}$. À bien regarder les pièces de Bärfuss toutefois, on discerne une forte présence du politique, qui n'est certes pas abordé frontalement - les œuvres de Bärfuss ne sauraient s'y résumer tant on y décèle d'autres enjeux, esthétiques notamment. Or même si le sujet des pièces semble ménager une place assez vaste à la réflexion politique ou sociétale (la fin de vie et ses enjeux éthiques, le regard sur la différence), une lecture approfondie nous montre que c'est bien plutôt dans les interstices du canevas que se manifestent et se discutent de telles problématiques.

Les débats soulevés ne sont en effet jamais engagés de manière abstraite ni didactique, mais à travers le destin singulier d'un person-

37. - Voir le récitatif du chœur, ibid.,p. 12.

38. - «Es gibt keine Anspruchshaltung an die Literatur».

39. - «Wie politisch darf Literatur sein? » Aus Echo der Zeit, 02.05. 2008. URL: http://www.srf.ch [consulté le 19.11.13]. 
nage qui, par le biais de son parcours - d'où l'importance poétologique du cheminement, évoquée au début de cette contribution - provoque une réflexion incarnée et humaine sur un thème contemporain et générateur de clivage au sein de la société, qu'il s'agisse de l'engagement littéraire et médiatique, du suicide assisté, de la foi ou des liens de filiation. L'objectif visé n'est nullement l'édification, et la posture de l'auteur est tout sauf moralisatrice. Bärfuss veille plutôt à opérer l'imbrication féconde de la sphère intime, individuelle, et d'un questionnement de nature plus vaste, plus politique et plus universelle. Sur son site Internet, la compagnie « 400asa » définit d'ailleurs en ces termes son travail : « Le collectif 400asa a toujours conçu son théâtre comme une entreprise politique, comique et rythmique $»^{40}$.

L'un des principaux enjeux pour la jeune génération de dramaturges helvétiques qui a émergé dans les années 1990 était probablement de se faire une place et de se trouver une identité propre après l'hégémonie de Frisch et de Dürrenmatt. Certains, comme par exemple Stefan Kaegi, ont opté pour le genre du théâtre documentaire. Lukas Bärfuss a pour sa part choisi de maintenir une trame dramatique, sans rejeter la narrativité ni la linéarité. En ce sens, Bärfuss est bien un raconteur d'histoires, et c'est un statut qu'il assume, $y$ compris dans un pays où il est devenu si difficile d'en raconter ${ }^{41}$. Mais il veille malgré tout à tisser des canevas suffisamment lâches pour ménager de la place à une réflexion de nature tant politique qu'esthétique. À l'occasion de cette contribution, nous souhaitions mettre en avant dans les pièces de Lukas Bärfuss auxquelles nous avons consacré notre analyse l'importance du cheminement, du parcours, ainsi que du terme dramatique, lequel se révèle n'être qu'un seuil vers la poursuite de la réflexion, et non une clôture. C'est d'ailleurs probablement pourquoi, chez Lukas Bärfuss, les pellicules se dévident plutôt que les rideaux, à l'instar des sentences, ne tombent.

40. - « Die Gruppe 400asa wollte und will ihr Theater immer politisch, komisch und rhythmisch ». URL: http://400asa.ch/400asa/geschichte.php [consulté le 03.12.13].

41. - Nous renvoyons ici aux travaux de Rosmarie Zeller au sujet du roman contemporain en Suisse, marqué du sceau de «l'inénarrabilité du monde moderne ». 


\section{Bibliographie}

\section{Sources :}

Bärfuss, Lukas : Stücke, Göttingen, Wallstein Verlag, 2005.

Bärfuss, Lukas : Stücke, Göttingen, Wallstein Verlag, 2007.

\section{Littérature secondaire :}

Brender, Edwige, Hausbei, Kerstin, et al. (dir.) : À la croisée des langages. Texte et art dans les pays de langue allemande, Paris, Presses Sorbonne nouvelle, 2006.

Lehmann, Hans-Thies : Postdramatisches Theater, Frankfurt am Main, Verlag der Autoren, 1999.

Poschmann, Gerda: Der nicht mehr dramatische Theatertext: aktuelle Bühnenstücke und ihre dramaturgische Analyse. Tübingen, Niemeyer, 1997.

Tigges, Stefan (dir.): Dramatische Transformationen. Zu gegenwärtigen Schreibund Aufführungsstrategien im deutschsprachigen Theater, Bielefeld, transcript, 2008.

Zeller, Rosmarie: Der neue Roman in der Schweiz. Die Unerzählbarkeit der modernen Welt. Freiburg, Universitätsverlag, 1992. 